\title{
Asymptotic-Information-Lossless Designs and Diversity-Multiplexing tradeoff
}

\author{
Shashidhar V \\ ECE Department \\ Indian Institute of Science \\ Bangalore - 560012 INDIA \\ Email: shashidhar@protocol.ece.iisc.ernet.in
}

\author{
B. Sundar Rajan \\ ECE Department \\ Indian Institute of Science \\ Bangalore - 560012 INDIA \\ Email: bsrajan@ece.iisc.ernet.in
}

\author{
P. Vijay Kumar \\ ECE Department \\ Indian Institute of Science \\ Bangalore - 560012 INDIA \\ Email:vijay@ece.iisc.ernet.in
}

\begin{abstract}
It is well known that in the Zheng-Tse optimal diversity-multiplexing tradeoff curve, the Alamouti scheme meets the point corresponding to the maximum diversity gain only, whereas V-BLAST meets only the point corresponding to the maximum multiplexing gain. In this paper, we define AsymptoticInformation-Lossless (AILL) designs and obtain a necessary and sufficient condition under which a design is AILL. Analogous to the condition that full-rank designs achieve the point corresponding to the zero multiplexing gain of the optimal tradeoff, we show that it is a necessary and sufficient condition for a design to be AILL to achieve the point corresponding to the zero diversity gain of the optimal tradeoff curve. Also, we obtain a lower bound on the tradeoff achieved by the designs from field extensions and division algebras. The lower bound for the designs from division algebras indicates that they achieve both the extreme points (corresponding to the zero diversity gain and zero multiplexing gain) of the optimal tradeoff curve.
\end{abstract}

\section{INTRODUCTION AND PRELIMINARIES}

A space-time code for $n_{t}$ transmit antennas is a finite set of $n_{t} \times l$ matrices (called codewords), where $l$ is an integer such that the channel does not change for an integral multiple of $l$ symbol intervals. If $\mathbf{X} \in \mathcal{C}$ is the codeword transmitted, where $\mathcal{C}$ is an STBC and the number of receive antennas is $n_{r}$, we have

$$
\mathbf{Y}=\sqrt{\frac{\mathrm{SNR}}{n_{t}}} \mathbf{H X}+\mathbf{W}
$$

where $\mathbf{Y} \in \mathbb{C}^{n_{r} \times l}$ is the received matrix, $\mathbf{H} \in \mathbb{C}^{n_{r} \times n_{t}}$ is the channel matrix and $\mathbf{W} \in \mathbb{C}^{n_{r} \times l}$ is the additive noise both with entries independent complex Gaussian with zero mean and unit variance. Under the assumption that $\mathcal{E}\left[\operatorname{tr}\left(\mathbf{X X}^{*}\right)\right]=n_{t} l$, the average signal-to-noise (SNR) at each receive antenna is SNR. At high SNRs, the pair-wise error probability that the received matrix $\mathbf{Y}$ is decoded to a codeword matrix $\mathbf{X}^{\prime} \neq \mathbf{X}$ is

$$
P\left(\mathbf{X} \rightarrow \mathbf{X}^{\prime}\right) \propto \mathrm{SNR}^{-n_{r} \Lambda}
$$

where $\Lambda$ is the rank of $\Delta=\mathbf{X}-\mathbf{X}^{\prime}$. The gain due to the spatial diversity provided by $n_{t}$ transmit and $n_{r}$ receive antennas is, quantitatively, termed as diversity gain, $d$, and is equal to $n_{r} \Lambda$.

\footnotetext{
${ }^{1}$ This work was partly funded by the IISc-DRDO Program on Advanced Research in Mathematical Engineering through a grant to B.S.Rajan and in part by an NSF-ITR Grant 0326628 to P. Vijay Kumar.
}

Any STBC with diversity gain equal to $n_{t} n_{r}$ is called a fullrank STBC. References [1]-[7], [9]-[12] provide some of the well known full-rank STBCs.

Another advantage due to the multiple transmit and receive antennas is based on capacity analysis of the channel. At high SNRs, the capacity of a Rayleigh fading channel with $n_{t}$ transmit and $n_{r}$ receive antennas is

$$
C\left(n_{t}, n_{r}, \mathrm{SNR}\right)=\min \left\{n_{t}, n_{r}\right\} \log \mathrm{SNR}+O(1) .
$$

The above expression shows that the achievable data rate increases with SNR as $\min \left\{n_{t}, n_{r}\right\} \log \mathrm{SNR}$. Since, this value is $\min \left\{n_{t}, n_{r}\right\}$ times the capacity of a single-antenna channel, a multiple-antenna channel can be viewed as $\min \left\{n_{t}, n_{r}\right\}$ parallel channels and hence $\min \left\{n_{t}, n_{r}\right\}$ is the total number of degrees of freedom (independent information symbols that can be communicated). This advantage due to the multiple antennas is called spatial multiplexing [15], [16].

It has been shown recently in [17], that both diversity and spatial multiplexing can be obtained simultaneously but with a fundamental tradeoff between them. Let $\mathcal{C}(S N R)$ be the code corresponding to the SNR level SNR and the data rate in bits per channel use achieved by $\mathcal{C}(\mathrm{SNR})$ be $R(\mathrm{SNR})$. Then, the set of all codes $\{\mathcal{C}(\mathrm{SNR})\}$ is called a scheme.

Definition 1 ([17]): A scheme $\{\mathcal{C}(\mathrm{SNR})\}$ is said to achieve a spatial multiplexing gain $r$ and a diversity gain $d$ if

$$
\lim _{\mathrm{SNR} \rightarrow \infty} \frac{R(\mathrm{SNR})}{\log \mathrm{SNR}}=r \text { and } \lim _{\mathrm{SNR} \rightarrow \infty} \frac{\log P_{e}}{\log \mathrm{SNR}}=-d
$$

where $P_{e}$ is the error probability.

Following the notation of [17], we use $\doteq$ for exponential equality. Thus, $2^{R(\mathrm{SNR})} \doteq \mathrm{SNR}^{r}$ and $P_{e} \doteq \mathrm{SNR}^{-d}$. Similarly, we use $\geq$ and $\dot{\leq}$ for exponential inequalities.

Definition 2: A rate- $k / l, n \times l$ design over a subfield $K$ of the complex field $\mathbb{C}$, is an $n \times l$ matrix $\mathbf{M}\left(x_{1}, x_{2}, \ldots, x_{k}\right)$ with entries $K$-linear combinations of $k$ variables and their conjugates. We call $\mathbf{M}$ a full-rank design over a field $F$ if every finite subset of the set $E=\left\{\mathbf{M}\left(x_{1}, x_{2}, \ldots, x_{k}\right) \mid x_{i} \in\right.$ $F, i=1,2, \ldots, k\}$, is a full-rank STBC.

Restricting the variables $x_{i}$ to come from a finite subset, called the signal set or constellation, of the field $F$, we get a fullrank STBC. Notice that though $x_{i}$ come from a signal set, the 
complex symbols that are transmitted can be from a different set. By changing the signal sets such that their size increases with SNR we get a scheme. Thus, a scheme can be described by a design and a class of signal sets.

Zheng and Tse in [17] have obtained lower and upper bound on the optimal diversity-multiplexing tradeoff curve. The upper bound is given by the exponent $d_{\text {out }}$ of the outage probability. The lower bound for the case $l \geq n_{t}+n_{r}-1$ is again given by $d_{\text {out }}$. Hence, for the case $l \geq n_{t}+n_{r}-1$, the optimal diversity-multiplexing tradeoff is $d(r)=d_{\text {out }}(r)=$ $\left(n_{t}-r\right)\left(n_{r}-r\right)$. For the case $l<n_{t}+n_{r}-1$, the lower bound does not match with the upper bound and hence there is no exact expression for the optimal tradeoff. When $n_{t}=n_{r}=2$ and $l \geq 2$, however, it has been proved that the lower bound and the upper bound coincide [18].

We say a design achieves the optimal tradeoff if there exists a class of signal sets, one for each SNR level, such that the scheme described by the design and the class of signal sets achieves the optimal tradeoff. If a scheme is described by a full-rank design over a field $F$ and a class of signal sets which are subsets of $F$, then the maximum diversity gain achieved by such a scheme is the diversity gain of any code in the scheme. Schemes like V-BLAST [16] achieve the point $\left(\min \left\{n_{t}, n_{r}\right\}, 0\right)$ of the optimal tradeoff curve. In [18], a scheme for 2 transmit and 2 receive antennas was constructed which achieves the optimal tradeoff for all $0 \leq r \leq 2$.

In this paper, we define asymptotic-information-lossless STBCs and show that asymptotic-information-losslessness is a necessary condition for achieving the optimal diversitymultiplexing tradeoff. Using the notion of asymptoticinformation-losslessness, we discuss the tradeoff achievability of some well known codes like STBCs from OD, QOD and Linear Dispersion codes. We obtain lower bound on the diversity-multiplexing tradeoff for the STBCs obtained from field extensions [6] and non-commutative division algebras [6], [7]. The lower bound for STBCs from division algebras indicates that these codes achieve the points $\left(0, n_{t} n_{r}\right)$ and $\left(\min \left\{n_{t}, n_{r}\right\}, 0\right)$ of the optimal tradeoff curve.

\section{ASYMPTOTIC-INFORMATION-LOSSLESS DESIGNS}

In this section, we define Asymptotic-Information-Lossless (AILL) Designs and show that it is a necessary condition for achieving the optimal diversity-multiplexing tradeoff. We also give a sufficient condition under which a design is AILL.

Let $\mathbf{X}$ be a rate- $k / l, n_{t} \times l$ design with $a_{1}, a_{2}, \ldots, a_{k}$ as the variables. If $\mathbf{X}$ is the design used to describe an STBC, then the received matrix is $\mathbf{Y}$ as in (1). Suppose, we can rewrite (1) as

$$
\tilde{\mathbf{y}}=\sqrt{\frac{\mathrm{SNR}}{n_{t}}} \widehat{\mathbf{H}} \tilde{\mathbf{a}}+\tilde{\mathbf{w}}
$$

where $\tilde{\mathbf{a}}$ is a $k \times 1$ vector containing either $a_{i}$ or $a_{i}^{*}$ but not both $a_{i}$ and $a_{i}^{*}$. Then, we define the capacity of the design $\mathbf{X}$ as the capacity of the equivalent channel $\tilde{\mathbf{H}}$ given by

$$
C_{\mathbf{X}}\left(n_{t}, n_{r}, \mathrm{SNR}, \mathbf{H}\right)=\frac{1}{l} \log _{2}\left(\operatorname{det}\left(\mathbf{I}_{n_{r} l}+\frac{\mathrm{SNR}}{n_{t}} \widehat{\mathbf{H}} \widehat{\mathbf{H}}^{\dagger}\right)\right) \text {. }
$$

Clearly, $C_{\mathbf{X}}\left(n_{t}, n_{r}, \mathrm{SNR}\right):=\mathcal{E}_{\mathbf{H}}\left[C_{\mathbf{X}}\left(n_{t}, n_{r}, \mathrm{SNR}, \mathbf{H}\right)\right] \leq$ $C\left(n_{t}, n_{r}, \mathrm{SNR}\right)$. If $\mathbf{X}$ has entries which are functions of the variables $a_{i}, i=1,2, \ldots, k$ and not of their conjugates, then we can rewrite (1) as

$$
\mathbf{y}=\sqrt{\frac{\mathrm{SNR}}{n_{t}}} \underbrace{\left[\begin{array}{cccc}
\mathbf{H} & \mathbf{0} & \cdots & \mathbf{0} \\
\mathbf{0} & \mathbf{H} & \cdots & \mathbf{0} \\
\vdots & \vdots & \cdots & \vdots \\
\mathbf{0} & \mathbf{0} & \cdots & \mathbf{H}
\end{array}\right]}_{\mathcal{H}} \underbrace{\left[\begin{array}{c}
\mathbf{X}_{1} \\
\mathbf{X}_{2} \\
\vdots \\
\mathbf{X}_{l}
\end{array}\right]}_{\mathbf{x}}+\mathbf{w} .
$$

where $\mathbf{y}$ and $\mathbf{w}$ are the column vectors obtained by serializing the columns of $\mathbf{Y}$ and $\mathbf{W}$ respectively and $\mathbf{X}_{i}$ denotes the $i$-th column of $\mathbf{X}$. With $\Phi$ as an $n_{t} l \times k$ matrix such that $\Phi \mathbf{a}=\mathbf{x}$ where $\mathbf{a}=\left[\begin{array}{llll}a_{1} & a_{2} & \ldots & a_{k}\end{array}\right]^{T}$, we have

$$
\mathbf{y}=\sqrt{\frac{\mathrm{SNR}}{n_{t}}} \underbrace{\mathcal{H} \Phi}_{\widehat{\mathbf{H}}} \mathbf{a}+\mathbf{w}
$$

We call the matrix $\Phi$ as generator of $\mathbf{X}$.

Definition 3: We call $\mathbf{X}$ an asymptotic-information-lossless (AILL) design for $n_{r}$ receive antennas if

$$
\lim _{\mathrm{SNR} \rightarrow \infty} \frac{C\left(n_{t}, n_{r}, \mathrm{SNR}\right)}{C_{\mathbf{X}}\left(n_{t}, n_{r}, \mathrm{SNR}\right)}=1 .
$$

The design $\mathbf{X}$ is called an information-lossless (ILL) design for $n_{r}$ receive antennas if $C\left(n_{t}, n_{r}, \mathrm{SNR}\right)=$ $C_{\mathbf{X}}\left(n_{t}, n_{r}\right.$, SNR $) \quad \forall$ SNR [6]-[8], [13] and have been well studied.

Theorem 1: The design $\mathbf{X}$ is AILL design for $n_{r}$ receive antennas if and only if the matrix $\widehat{\mathbf{H}}$ has rank at least $\min \left\{n_{t}, n_{r}\right\} \times l$.

Since, the rank of the matrix $\Phi$ is bounded above by $k$, the number of variables in the design, and if the design is AILL, then it is necessary but not sufficient that $k \geq l \times \min \left\{n_{t}, n_{r}\right\}$. The following example gives a design which satisfies the necessary condition but still fails to be AILL.

Example 1: Let $n_{t}=2$. Let $\mathbf{X}$ be the design $\left[\begin{array}{cc}a_{0}+z a_{1} & \delta\left(a_{2}+z a_{3}\right) \\ a_{2}+z a_{3} & a_{0}+z a_{1}\end{array}\right]$ where $z$ and $\delta$ are two independent transcendental elements over the rational number field $\mathbb{Q}$. Clearly, the number of variables in the design $\mathbf{X}$ is $k=4$ and hence the design satisfies the necessary condition $k \geq$ $l \times \min \left\{n_{t}, n_{r}\right\}$. The generator matrix $\Phi$ of this design is $\left[\begin{array}{cccc}1 & z & 0 & 0 \\ 0 & 0 & 1 & z \\ 0 & 0 & \delta & \delta z \\ 1 & z & 0 & 0\end{array}\right]$. Clearly, the rank of $\Phi$ is 2 and hence from Theorem 1, this design is asymptotic-information-lossy (AIL) for 2 receive antennas. However, it is AILL for 1 receive antenna.

It can be checked that except the Alamouti code, all other complex ODs (CODs) are AIL for any number of receive antennas. The $2 \times 2 \mathrm{COD}$ is AILL for 1 receive antenna and AIL for $n_{r} \geq 2$ receive antennas. Similarly, the QODs and CIODs [3] for $n_{t}=2,3,4$ are AILL for 1 receive antenna and AIL for $n_{r} \geq 2$ receive antennas. All other QODs and CIODs are AIL for any number of receive antennas. 
In [20], codes called Linear Dispersion (LD) codes were constructed to obtain maximum mutual information. The number of variables was chosen to be $\min \left\{n_{t}, n_{r}\right\} \times l$. It can be checked easily that for all the LD codes presented in [20], the rank of the matrix $\widehat{\mathbf{H}}$ is equal to $\min \left\{n_{t}, n_{t}\right\} \times l$ and hence all the LD codes of [20] are AILL designs. Most of the well known designs like DAST [10], designs from field extensions [6] are AILL for 1 receive antenna.

Corollary 1: Let $\mathbf{X}$ be a rate- $k / l, n_{t} \times l$ AILL design for any number of receive antennas. Then, $k \geq n_{t} l$.

Example 2: Let $n_{t}=2$. Let $\mathbf{X}$ be the design $\left[\begin{array}{cc}a_{0}+a_{1} \sqrt{j} & \delta\left(a_{2}-a_{3} \sqrt{j}\right) \\ a_{2}+a_{3} \sqrt{j} & a_{0}-a_{1} \sqrt{j}\end{array}\right]$ where $\delta$ is a transcendental element over $\mathbb{Q}$. The generator matrix $\Phi$ is $\left[\begin{array}{cccc}1 & \sqrt{j} & 0 & 0 \\ 0 & 0 & 1 & \sqrt{j} \\ 0 & 0 & \delta & -\delta \sqrt{j} \\ 1 & -\sqrt{j} & 0 & 0\end{array}\right]$. It is easy to check that the rank of the matrix $\Phi$ is 4 and hence is AILL for any number of receive antennas. It can be checked that this design is, in fact, an Information lossless design. This design is same as the design obtained in [9] with $\delta=1+2 j$, in which case the design is not ILL but continues to be AILL.

Analogous to the sufficient condition that full-rank designs achieve the point corresponding to the zero multiplexing gain of the optimal diversity-multiplexing tradeoff, we now give a necessary and sufficient condition for any design to achieve the point corresponding to the zero diversity gain of the optimal diversity-multiplexing tradeoff. With $R=r \log$ SNR as the data rate we have the codeword error probability given by

$P_{e}(\mathrm{SNR}) \doteq P_{\text {out }, \mathbf{X}}(r)+P($ error $\mid$ no outage $) \geq P_{\text {out }}(r)$

where $P_{\text {out }, \mathbf{X}}(r)=P\left(C_{\mathbf{X}}\left(n_{t}, n_{r}, \mathrm{SNR}\right) \leq r \log \mathrm{SNR}\right)$. Clearly, $P_{\text {out }, \mathbf{X}}(r)$ is greater than or equal to the channel outage probability $P_{\text {out }}(r)$ given by $P\left(C\left(n_{t}, n_{r}, \mathrm{SNR}\right) \leq R\right)$. From (3) note that

$$
d_{\mathbf{X}}(r) \leq d_{\text {out }, \mathbf{X}}(r) \leq d_{\text {out }}(r)
$$

where $P_{\text {out }, \mathbf{X}}(r) \doteq \mathrm{SNR}^{-d_{\text {out }}, \mathbf{x}(r)}$ and $P_{\text {out }}(r) \doteq$ $\mathrm{SNR}^{-d_{\text {out }}(r)}$. This tells us that the optimal diversitymultiplexing tradeoff curve is upper bounded by $d_{\text {out }}(r)$ [17] .

Theorem 2: Let $\mathbf{X}$ be a rate- $k / l, n_{t} \times l$ design which achieves optimal diversity-multiplexing tradeoff for $n_{r}$ receive antennas. Then, $\mathbf{X}$ is an AILL design for $n_{r}$ receive antennas. In other words asymptotic information-losslessness is a necessary condition for a design to achieve the optimal diversitymultiplexing tradeoff.

Proof: Follows from Chebyshev's inequality.

We now show that AILL is also a sufficient condition for a design to achieve the point $\left(0, \min \left\{n_{t}, n_{r}\right\}\right)$ of the tradeoff curve.

Theorem 3: The design $\mathbf{X}$ achieves the point $\left(\min \left\{n_{t}, n_{r}\right\}, 0\right)$ of the diversity-multiplexing tradeoff curve if and only if $\mathbf{X}$ is an AILL design for $n_{r}$ receive antennas.
Proof: Let $\mathbf{X}$ be an AILL design for $n_{r}$ receive antennas. Then, we have two cases:

Case 1. $n_{r} \geq n_{t}$. Then, the generator matrix $\Phi$ of $\mathbf{X}$ has rank at least $n_{t} l$. Let the singular value decomposition $\Phi$ be $\Phi=\mathbf{U D V}^{\dagger}$. Then, the equivalent system model for the design $\mathbf{X}$ is

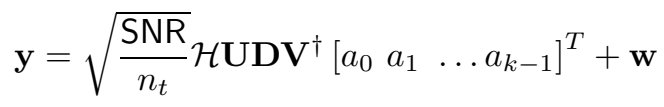

where $k$ is the number of variables in $\mathbf{X}$ and $a_{i}$ are the variables. If $k>n_{t} l$, we allow only $n_{t} l$ of the $k$ variables to take values from some constellation, while restrict the remaining to zero. Thus, without loss of generality let $k=n_{t} l$. To obtain the error probability, let us restrict ourselves to QAM signal constellations. If the data rate $R=r \log \mathrm{SNR}$, then $a_{i}$ should take values from SNR ${ }^{l r / k}$-QAM. If $d_{\min }$ is minimum Euclidean distance of the $\mathrm{SNR}^{l r / k}$-QAM constellation, then the minimum Euclidean distance of the constellation of the vectors $\mathbf{U D V}{ }^{\dagger} \mathbf{x}$ is at least $\lambda_{\min } d_{\min }$, where $\lambda_{\min }$ is the minimum among all the non-zero diagonal elements of the diagonal matrix $\mathbf{D}$. Also, it is easy to see that the number of nearest neighbors in the constellation $\mathrm{UDV}^{\dagger} \mathbf{x}$ is a constant. Thus, we can view the system now as

$$
\mathbf{y}=\sqrt{\frac{S N R}{n_{t}}} \mathcal{H} \widehat{\mathbf{x}}+\mathbf{w}
$$

where $\widehat{\mathbf{x}}=\mathbf{U D V}^{\dagger} \mathbf{x}$. Now, entries of $\widehat{\mathbf{x}}$ come from a constellation whose minimum distance is greater than or equal to $\lambda_{\min } d_{\min }$ and the number of nearest neighbors is a constant independent of SNR. Using the technique of successive nulling and cancelling of V-BLAST, we have the equivalent system of the channel as

$$
\mathbf{y}_{i}=\sqrt{\frac{\mathrm{SNR}}{n_{t}}} g_{i} \widehat{\mathbf{x}}_{i}+w_{i} \quad i=0,1,2, \ldots, n_{t} l
$$

where $g_{i}^{2}$ is the $i$-th decorrelator SNR gain and is a chi-squared distributed random variable with $2 j$ degrees of freedom where $j=i \bmod n_{r}$. From [17], the pairwise error probability for $i$-th decorrelator is

$$
P_{e}\left(x_{i} \rightarrow x_{i}^{\prime}\right) \doteq P\left(\frac{\mathrm{SNR}}{n_{t}} g_{i}^{2}\left\|x_{i}-x_{i}^{\prime}\right\|^{2}<1\right)
$$

Since the minimum squared Euclidean distance of the $\mathrm{SNR}^{l r / k}$-QAM is equal to $\mathrm{SNR}^{-l r / k}$, we have

$$
P_{e}\left(x_{i} \rightarrow x_{i}^{\prime}\right) \doteq P\left(g_{i}^{2}<\frac{n_{t}}{\mathrm{SNR}^{1-l r / k}}\right) \doteq \mathrm{SNR}^{-(1-l r / k)} .
$$

Since, the number of nearest numbers is a constant independent of SNR, the actual error probability for $i$-th decorrelator is

$$
P_{e}^{(i)}(\mathrm{SNR}) \doteq \mathrm{SNR}^{-(1-l r / k)} .
$$

Since $P_{e}^{(1)}(\mathrm{SNR}) \leq P_{e}^{\prime}(\mathrm{SNR}) \leq \sum_{i} P_{e}^{(i)}(\mathrm{SNR})$, where $P_{e}^{\prime}(\mathrm{SNR})$ is the error probability with successive nulling and cancelling detection, we have the actual error probability with ML detection given by

$$
P_{e}(\mathrm{SNR}) \leq P_{e}^{\prime}(\mathrm{SNR}) \doteq \mathrm{SNR}^{-(1-l r / k)} .
$$


Since, $\mathbf{X}$ is an AILL design, we have $l / k=1 / n_{t}$. Hence, $d_{\mathbf{X}}(r)$ is equal to zero when $r=n_{t}$, i.e., the point corresponding to zero diversity is $\left(n_{t}, 0\right)$.

Case 2. $n_{t} \geq n_{r}$. In this case, we can assume that the design has $n_{r} l$ variables. Now, the matrix $\mathcal{H} \Phi$ is a $n_{r} l \times n_{r} l$ matrix with entries as linear combinations of the entries of $\mathbf{H}$. Hence, the entries of the matrix $\mathcal{H} \Phi$ are Gaussian distributed and the rank of $\mathcal{H} \Phi$ is $n_{r} l$. Following the method given in Case 1., we have the probability of error upper bounded by

$$
P_{e}(\mathrm{SNR}) \dot{\leq} \mathrm{SNR}^{-\left(1-r / n_{r}\right)}
$$

and hence the point $\left(n_{r}, 0\right)$ of the optimal tradeoff curve is achieved.

Since in any $n_{t} \times l$ orthogonal design $\left(n_{t} \neq 2\right) \mathbf{X}$, the number of the variables is strictly less than $l, \mathbf{X}$ is AIL and hence from the above theorem, all the orthogonal designs except Alamouti scheme do not achieve the optimal tradeoff for any number of receive antennas. Alamouti scheme has been shown to achieve the optimal tradeoff for 1 receive antenna. Similarly, QODs for $n_{t} \geq 5$ do not achieve the optimal tradeoff for any number of receive antennas. For $n_{t}=2,3,4$, the QODs achieve the point $(0,1)$ of the tradeoff curve for 1 receive antenna.

Corollary 2: If $\mathbf{X}$ is a full-rank design for $n_{t}$ transmit antennas, such that the STBCs constructed using the design $\mathbf{X}$ are completely over a signal set $S$, i.e., entries of the codeword matrices in the STBC are from the signal set $S$ over which the STBC is constructed, then the design $\mathbf{X}$ does not achieve the point corresponding to the zero diversity gain of the tradeoff curve for more than one receive antenna.

Remark 1: Let $\mathbf{X}$ be an ILL design and $\mathbf{X}^{\prime}$ be an AILL design but not ILL. Suppose, both $\mathbf{X}$ and $\mathbf{X}^{\prime}$ achieve the optimal diversity-multiplexing tradeoff. Then, clearly, it is preferable to use the design $\mathbf{X}$ to the design $\mathbf{X}^{\prime}$ because the former is ILL while the later is not ILL. So, it is important to construct designs which not only achieve optimal diversitymultiplexing tradeoff, but also are ILL.

\section{Diversity-MultipleXing Tradeoff of Designs FROM FIELD EXTENSIONS}

Let $\mathbf{X}$ be a rate- $k / n_{t}, n_{t} \times n_{t}$ design obtained from the field extension of $\mathbb{Q}\left(\omega_{m}\right)$ using a minimal polynomial of the form $x_{t}^{n}-\omega_{m}$, where $\omega_{m}=e^{j 2 \pi / m}$ and $m$ is a positive integer such that the set of the prime factors of $m$ contains the set of prime factors of $n_{t}$ [6]. Then, the design $\mathbf{X}$ is of the form

$$
\left[\begin{array}{cccc}
f_{0} & \omega_{m} f_{n_{t}-1} & \cdots & \omega_{m} f_{1} \\
f_{1} & f_{0} & \cdots & \omega_{m} f_{2} \\
\vdots & \vdots & \ddots & \vdots \\
f_{n_{t}-1} & f_{n_{t}-2} & \cdots & f_{0}
\end{array}\right]
$$

It can be checked that the generator matrix of this design has rank $n_{t}$. Thus, $\mathbf{X}$ is an AILL design only for 1 receive antenna. From Theorem 3, the diversity-multiplexing tradeoff of this design for 1 receive antenna is lower bounded by $d(r)=1-r$ for $0<r \leq 1$ and since the design is a full-rank design over the field $\mathbb{Q}\left(\omega_{m}\right)$, we also have $d(0)=n_{t}$. Notice that the lower bound we have from Theorem 3 when used for the designs from field extensions is independent of the number of transmit and receive antennas. Further, with the assumption that the determinants of $\mathbf{X}-\mathbf{X}^{\prime}$ are uniformly distributed we have a stronger lower bound given by

$$
d_{\mathbf{X}}(r) \geq n_{t} n_{r}(1-r)-r\left(n_{t}-1\right)
$$

Figure 1 shows the tradeoff curve for $n_{t}=2$ and $n_{r}=1,2$.
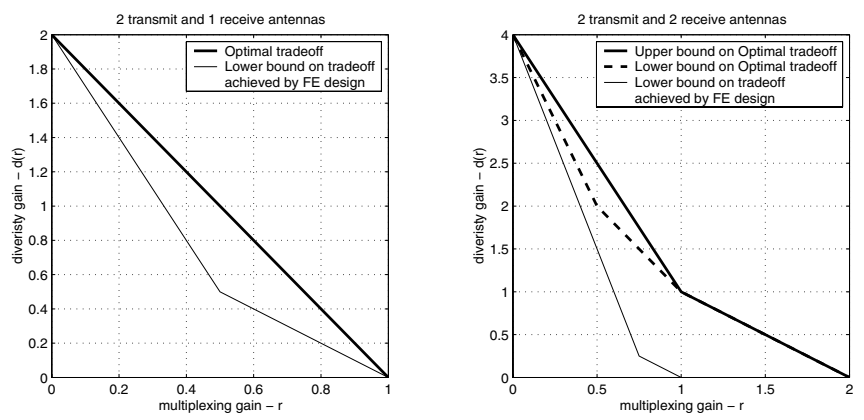

Fig. 1. Diversity-multiplexing tradeoff achieved by design from field extensions for 2 transmit and 1,2 receive antennas

\section{Diversity-Multiplexing Tradeoff of Designs FROM DIVISION ALGEBRA}

In this section, we briefly describe the codes obtained in [6], [7] and then give a lower bound on the tradeoff.

Let $S$ be the signal set (a finite subset of $\mathbb{C}$ ) over which we want to construct full-rank STBCs. Let $m$ be an integer such that $x^{n}-\omega_{m}$ is irreducible over $F=\mathbb{Q}\left(S, \omega_{m}\right)$, where $\omega_{m}=$ $e^{j 2 \pi / m}$. The extension field obtained from this irreducible polynomial is $K=F\left(\omega_{m n}\right) \supset F=\mathbb{Q}\left(S, \omega_{m}\right) \supset S$. Then, we have the following theorem.

Theorem 4 ([6], [7]): Let $G$ be the Galois group of the extension $K / F$. Clearly, $G$ is cyclic and let $\sigma$ be a generator of this group. Let $\delta$ be a transcendental element over $F$. Then, the set $D$ of matrices of the form as in (4), where $t=\omega_{m n}$ and $f_{i, j} \in F(\delta)$ for $i, j=0,1, \ldots, n-1$ is a cyclic division algebra.

From the above theorem, restricting the variables $f_{i, j}$ to the signal set $S$, we have a full-rank STBC over $S$, for $n$ transmit antennas. Since the number of variables in the matrix of (4) is $n^{2}$, if the size of the signal set $S$ as a function of SNR is equal to $\mathrm{SNR}^{r / n}$, the bit rate of the code in bits per channel use will be $\frac{1}{n} \log _{2}\left(\mathrm{SNR}^{r / n}\right)^{n^{2}}=r \log \mathrm{SNR}$.

Example 3: Let $n=2$ and $S$ be a QAM signal set. Then, we take $F=\mathbb{Q}(j), K=F(\sqrt{j})$. Clearly, $K$ is the splitting field of the polynomial $x^{2}-j \in F[x]$ and hence $K / F$ is cyclic of degree 2. The generator of the Galois group is given by $\sigma: \sqrt{j} \mapsto-\sqrt{j}$. Now, let $\delta$ be any transcendental element over $F$. Then, we have a full-rank STBC $\mathcal{C}(\mathrm{SNR})$ with codewords

$$
\frac{1}{\sqrt{2}}\left[\begin{array}{cc}
f_{0,0}+f_{0,1} \sqrt{j} & \delta\left(f_{1,0}-f_{1,1} \sqrt{j}\right) \\
f_{1,0}+f_{1,1} \sqrt{j} & \left(f_{0,0}-f_{0,1} \sqrt{j}\right)
\end{array}\right]
$$




$$
\frac{1}{\sqrt{n}}\left[\begin{array}{ccccc}
\sum_{i=0}^{n-1} f_{0, i} t^{i} & \delta \sigma\left(\sum_{i=0}^{n-1} f_{n-1, i} t^{i}\right) & \delta \sigma^{2}\left(\sum_{i=0}^{n-1} f_{n-2, i} t^{i}\right) & \cdots & \delta \sigma^{n-1}\left(\sum_{i=0}^{n-1} f_{1, i} t^{i}\right) \\
\sum_{i=0}^{n-1} f_{1, i} t^{i} & \sigma\left(\sum_{i=0}^{n-1} f_{0, i} t^{i}\right) & \delta \sigma^{2}\left(\sum_{i=0}^{n-1} f_{n-1, i} t^{i}\right) & \cdots & \delta \sigma^{n-1}\left(\sum_{i=0}^{n-1} f_{2, i} t^{i}\right) \\
\vdots & \vdots & \vdots & \ddots & \vdots \\
\sum_{i=0}^{n-1} f_{n-1, i} t^{i} & \sigma\left(\sum_{i=0}^{n-1} f_{n-2, i} t^{i}\right) & \sigma^{2}\left(\sum_{i=0}^{n-1} f_{n-3, i} t^{i}\right) & \cdots & \sigma^{n-1}\left(\sum_{i=0}^{n-1} f_{0, i} t^{i}\right)
\end{array}\right]
$$

where $f_{i, j} \in \mathrm{SNR}^{r / 2} Q A M$. The data rate $R(\mathrm{SNR})$ is equal to $r \log$ SNR.

Theorem 5: The diversity-multiplexing tradeoff $d_{D A}(r)$ of the design from division algebras satisfies

$$
d_{D A}(r) \geq 1-r / \min \left\{n_{t}, n_{r}\right\} \text { and } d_{D A}(0)=n^{2} .
$$

If the number of receive antennas is 1 , then the tradeoff achieved by the designs from division algebras is same as that of achieved by the designs from field extensions.

Example 4 (Example 3 continued): The determinant of the design obtained in Example 3 is

$$
\operatorname{det} \mathbf{X}=N_{K / F}\left(f_{0,0}+\sqrt{j} f_{0,1}\right)-\delta N_{K / F}\left(f_{1,0}+\sqrt{j} f_{1,1}\right)
$$

where $N_{K / F}(x)$ is the algebraic norm of the element $x \in K$ from $K$ to $F$. Restricting the variables $f_{1,0}$ and $f_{1,1}$ to zero and assuming that the determinants of $\mathbf{X}-\mathbf{X}^{\prime}$ are uniformly distributed, we can tighten the lower bound on the tradeoff to obtain $d_{\mathbf{X}}(r) \geq 4-5 r$.
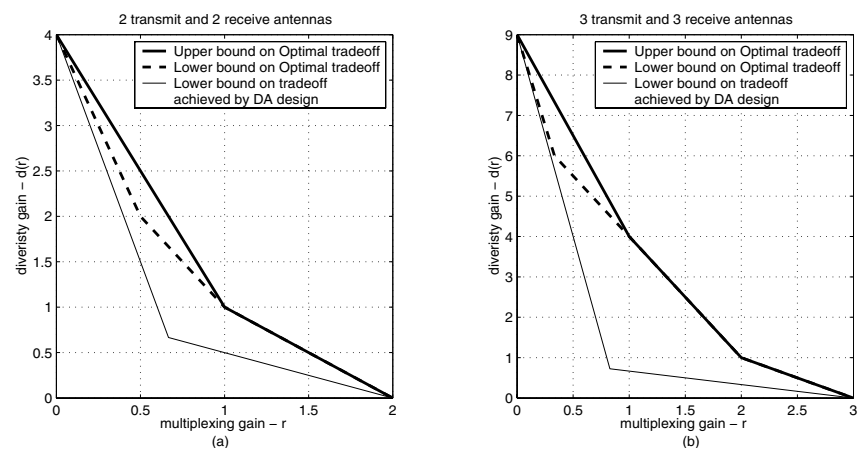

Fig. 2. Diversity-multiplexing tradeoff achieved by design from division algebras for (a) 2 transmit and 2 receive antennas, (b) 3 transmit and 3 receive antennas.

Figure 2 shows the lower bounds on the tradeoff achieved by the designs from division algebras for 2 and 3 transmit antennas. In [21], we have shown by simulations that the designs from division algebras achieve the optimal diversitymultiplexing tradeoff for $n_{t}=2,3,4$ and $n_{r}=n_{t}$.

\section{REFERENCES}

[1] Vahid Tarokh, H. Jafarkhani and A. R. Calderbank, "Space-Time block codes from orthogonal designs," IEEE Trans. Inform. Theory, vol.45, pp.1456-1467, July 1999 .

[2] O. Tirkonen and A. Hottinen, "Square-matrix embeddable space-time block codes for complex signal constellations," IEEE Trans. Inform. Theory, vol.48, no.2, Feb. 2002.

[3] Zafar Ali Khan and B. Sundar Rajan, " Space-time block codes from co-ordinate interleaved orthogonal designs," in Proc. IEEE Int. Symp. Inform. Theory (ISIT 2002), Lausanne, Switzerland, June 2002, p.275.
[4] Weifung-Su and Xiang-Gen Xia, "Quasi-orthogonal space-time block codes with full Diversity," in Proc. IEEE GLOBECOM, vol.2, 2002, pp.1098-1102.

[5] Olav Tirkkonen and Ari Hottinen, "Complex space-time block codes for four Tx antennas," in Proc. IEEE GLOBECOM, vol.2, 2000, pp.10051009.

[6] B. Sethuraman, B. Sundar Rajan and V. Shashidhar, "Full-diversity, highrate space-time block codes from division algebras," IEEE Trans. Inform. Theory: Special Issue on Space-Time Transmission, Reception, Coding and Signal Design, vol. 49, no. 10, pp. 2596-2616, Oct. 2003.

[7] V. Shashidhar, B. Sundar Rajan and B. A. Sethuraman,"STBCs using capacity achieving designs from cyclic division algebras", Proc. GLOBECOM 2003, Communication Theory Symposium, pp. 1957-1962, San Francisco, Dec.1-4, 2003.

[8] V. Shashidhar, B. Sundar Rajan and B. A. Sethuraman, "STBCs using capacity achieving designs from crossed-product division algebras", accepted for presentation in ICC 2004, Paris, France, June 20-24, 2004.

[9] J-C. Belfiore and G. Rekaya, "Quaternionic lattices for space-time coding", in Proc. IEEE Int. Workshop on Inform. Theory (ITW 2003), Paris, France, Mar.31 - Apr.4, 2003, pp.267-270.

[10] M. O. Damen, K. Abed-Meraim and J. -C. Belfiore, "Diagonal algebraic space-time block codes," IEEE Trans. Inform. Theory, vol.48, no.3, pp.628-636, Mar. 2002.

[11] J.Boutrous and E.Viterbo, "Signal Space Diversity : A power and bandwidth efficient diversity technique for the Rayleigh fading channel," IEEE Trans. Inform. Theory, vol.44, pp.1453-1467, jul 1998.

[12] Hesham El Gamal and M. O. Damen, "Universal space-time coding,", IEEE Trans. Inform. Theory, vol.49, no.5, pp.1097-1119, May 2003.

[13] M. O. Damen, Ahmed Tewfik and J. -C. Belfiore, "A construction of a space-time code based on number theory", IEEE Trans. Inform. Theory, vol.48, no.3, pp.753-760, Mar.2002.

[14] Hsiao-feng Lu and P. Vijay Kumar, "Rate-Diversity Tradeoff of SpaceTime Codes with Fixed Alphabet and Optimal Constructions," Proc. IEEE Int. Symp. Inform. Theory, Yokohoma, Japan, June 29 - July 4, 2003 , p. 242.

[15] R. Heath Jr. and A. Paulraj, "Switching between multiplexing and diversity based on constellation distance," in Proc. Allerton Conf. Communication, Control and Computing, Oct. 2000.

[16] G. J. Foschini, "Layered space-time architecture for wireless communications in a fading environment when using multi-element antennas," Bell Labs, Tech. J., Vol.1, No.2, pp.41-59, 1996.

[17] LiZhong Zheng, David N. C. Tse, "Diversity and multiplexing: A fundamental tradeoff in multiple-antenna channels", IEEE Trans. Inform. Theory, vol.49, no.5, pp.1073-1096, May 2003.

[18] Huan Yao and Gregory W. Wornell, "Structured space-time block codes with optimal diversity-multiplexing tradeoff and minimum delay", Proc. GLOBECOM 2003, Communication Theory Symposium, pp. 1941-1945, San Francisco, Dec.1-5, 2003.

[19] Hesham El Gamal, Giuseppe Caire, M. O. Damen, "Lattice coding and decoding achieve the optimal diversity-vs-multiplexing tradeoff of MIMO channels," submitted to Trans. Inform. Theory.

[20] B. Hassibi and B. Hochwald, "High-rate codes that are linear in space and time," IEEE Trans. Inform. Theory, vol.48, no.7, pp.1804-1824, July 2002.

[21] V. Shashidhar, B. Sundar Rajan and P. Vijay Kumar, "STBCs with optimal diversity-multiplexing tradeoff for 2, 3 and 4 transmit antennas," Proceedings of IEEE International Symposium on Information Theory (ISIT 2004), Chicago, June 27-July 2,2004 p 125. 